\title{
Spatially-variant directional mathematical morphology operators based on a diffused average squared gradient field
}

\author{
Rafael Verdú-Monedero ${ }^{1}$ and Jesús Angulo ${ }^{2}$ \\ 1 Department of Information Technologies and Communications, \\ Technical University of Cartagena, 30202, Cartagena, Spain, \\ rafael.verdu@upct.es \\ 2 Centre de Morphologie Mathématique, Ecole des Mines de Paris, \\ 35, rue Saint Honoré, 77305 Fontainebleau Cedex, France \\ jesus. angulo@ensmp.fr
}

\begin{abstract}
This paper proposes an approach for mathematical morphology operators whose structuring element can locally adapt its orientation across the pixels of the image. The orientation at each pixel is extracted by means of a diffusion process of the average squared gradient field. The resulting vector field, the average squared gradient vector flow, extends the orientation information from the edges of the objects to the homogeneous areas of the image. The provided orientation field is then used to perform a spatially variant filtering with a linear structuring element. Results of erosion, dilation, opening and closing spatially-variant on binary images prove the validity of this theoretical sound and novel approach.
\end{abstract}

\section{Introduction}

Mathematical morphology is nonlinear image processing methodology based on the application of lattice theory to spatial structures [1]. Typically, it relies on a probe set $B$, called structuring element, which is translated over each pixel of the image to compute a supremum/infimum operation. Morphological operators using line segments as structuring elements involve directional filtering effects. Recent advances in the fast implementation of morphological filters along discrete lines at arbitrary angles have been reported [2]. Other more sophisticated algorithms for morphological operators on thin structures are the path openings [3].

In this paper we focus on linear orientated structuring elements which are variable in the space according to a vector field. In mathematical morphology terms, that involves the notion of spatially-variant transformations. The corresponding theoretical aspects were already studied by Serra in [1], and more recent works deal with some advancements, see for instance [4]. The theoretical

\footnotetext{
* This work is partially supported by Ministerio de Ciencia y Tecnología, under grant TEC2006-13338/TCM
} 
framework of group morphology by Roerdink [5] deals also with non translation invariant morphology. Other papers focused specifically on the efficient implementation of spatially variant morphological operators are $[6,7]$.

This paper is organized as follows: Section 2 introduces spatially-variant binary morphology operators; Section 3 addresses the estimation of the orientation field and its diffusion process. Next, Section 4 contains some results of directional morphological operators on binary images, and finally conclusions close the paper.

\section{Spatially-variant binary morphological operators}

In the sequel we assume basic familiarity of the reader with the general concepts of mathematical morphology. General reference works are the books by Serra $[8$, 1], or the recent book by Soille [9].

\subsection{Background on spatial invariant mathematical morphology}

We remind in the section the most classical morphological operators for linear filtering and orientated structures.

A binary or Boolean image is considered as a set $X \subseteq E$, or more precisely as a function $X: E \rightarrow \mathcal{P}(E)$, where $\mathcal{P}(E)$ denotes the power set of $E$ comprising all subsets of $E$. The space support $E$ for the image domain can be the $d$-dimensional Euclidean space $\mathbb{R}^{d}$ or the equivalent discrete space $\mathbb{Z}^{d}$.

Choosing a fixed set, $B \subseteq E$, that one calls the structuring element, the two basic mappings $\mathcal{P}(E) \rightarrow \mathcal{P}(E)$ are the dilation by $B$, denoted by $\delta_{B}$, and given by

$$
\delta_{B}(X)=X \oplus \check{B}=\left\{\mathbf{h} \in E, X \cap B_{\mathbf{h}} \neq \emptyset\right\}=\bigcup_{\mathbf{b} \in \check{B}} X_{\mathbf{b}}=\bigcup_{\mathbf{x} \in X} \check{B}_{\mathbf{x}},
$$

and the erosion by $B$, denoted by $\varepsilon_{B}$ and defined by

$$
\varepsilon_{B}(X)=X \ominus \check{B}=\left\{\mathbf{h} \in E \mid B_{\mathbf{h}} \subseteq X\right\}=\bigcap_{\mathbf{b} \in \check{B}} X_{\mathbf{b}} .
$$

The structuring element is a subset defined in the origin $\mathbf{o} \in E$, then to each point $\mathbf{p}$ of $E$ corresponds the translation mapping $\mathbf{o}$ to $\mathbf{p}$, and this translation maps $B$ onto $B_{\mathbf{p}}$, i.e.,

$$
B_{\mathbf{p}}=\{\mathbf{b}+\mathbf{p}: \mathbf{b} \in B\} .
$$

We consider in the dilation and the erosion for a fixed structuring element $B$ all its translations, $B_{\mathbf{p}}$. This is because we assume that the space $E$ in which Boolean (or grey-level) images are represented is homogenous under the group of translations, also called translation invariant domain. In practice, one often chooses $B_{\mathbf{p}}=B_{\mathbf{p}} \cap E$ for $\mathbf{p} \in E$. In other words, the structuring element centered at point $\mathbf{p}$ is obtained by choosing a fixed structuring element and restricting the translations over the point $\mathbf{p}$ to points inside the "window" of $E$. Note that the 
dilation and the erosion are computed by using the set $\check{B}$, which is the reflection of $B$ with respect to the origin, i.e., $\check{B}=\{-\mathbf{b} \mid \mathbf{b} \in B\}$.

In general, it is not possible to recover the original image after dilation or erosion, i.e., morphological operators loss information. Starting from the adjunction pair $\left(\delta_{B}, \varepsilon_{B}\right)$, two operators are obtained. The (structural) Boolean opening by the structuring element $B$ is defined as

$$
\gamma_{B}(X)=(X \ominus \check{B}) \oplus B=\left\{\mathbf{h} \in E \mid \exists \mathbf{g} \in E, B_{\mathbf{g}} \subseteq X \text { and } \mathbf{h} \in B_{\mathbf{g}}\right\},
$$

or the more interesting expression:

$$
\gamma_{B}(X)=\bigcup_{\mathbf{h} \in E}\left\{B_{\mathbf{h}} \mid B_{\mathbf{h}} \subseteq X\right\},
$$

which allows to interpret the opening as the part of the space where the structuring element $B$ is totally included into $X$. The dual operator is the (structural) Boolean closing given by

$$
\varphi_{B}(X)=(X \oplus \check{B}) \ominus B=\left\{\mathbf{h} \in E \mid \mathbf{h} \in \check{B}_{\mathbf{y}} \Rightarrow \check{B}_{\mathbf{y}} \cap X \neq \emptyset\right\} .
$$

The binary closing has a dual interpretation for the complement.

In mathematical morphology, the structuring element is the shape probe which determines the effect of the operator. For the practical application of morphological filters, the autoreflected (or symmetrical) structuring elements, i.e., $B(\mathbf{x})=B(-\mathbf{x})$, are specially useful (and easier to implement). In particular, in Euclidean morphology, two main kinds of "shapes" are used as structuring elements: a centered circular ball (a disk) of radius $r$, denoted by $C^{r}$; and a centered line segment of length $l$ and orientation $\theta$, denoted by $L^{l, \theta}$. The structuring element $C^{r}$ is applied when isotropic effects are desired instead of $L^{l, \theta}$, more appropriate to achieve directional effects. For instance, the opening $\gamma_{L^{l, \theta}}(X)$ by the line segment of given slope $\theta$ and length $l$ will remove all white structures of $X$ except those having this orientation and at least this length. The dual operator $\varphi_{L^{l, \theta}}(X)$ removes black orientated structures of $X$.

Let $\gamma_{i}(X)$ be an opening for every $i \in I$, then $\bigvee_{i \in I} \gamma_{i}(X)$ is an opening as well. Let $\varphi_{i}(X)$ be a closing for every $i \in I$, then $\bigwedge_{i \in I} \varphi_{i}(X)$ is a closing as well. This property is useful to define derived directional operators. For instance, the linear opening of size $l$ is given by

$$
\gamma_{L^{l, \Theta}}(X)=\gamma_{L^{l, \theta_{1}}}(X) \vee \gamma_{L^{l, \theta_{2}}}(X) \vee \cdots \vee \gamma_{L^{l, \theta_{d}}}(X),
$$

where the following directions $\Theta=\left\{\theta_{1}, \theta_{2}, \cdots, \theta_{d}\right\}$ are considered and consequently, the opening $\gamma_{L^{l, \Theta}}(X)$ removes bright structures except those having these orientations and a length upper than $l$.

The top-hat (resp. dual top-hat) operator is very useful for extracting the structures which have been removed by an opening $\gamma$ (resp. a closing $\varphi$ ), and it is defined by the difference of both sets, i.e, $t h^{+}(X)=X \backslash \gamma(X)$ (resp. $t h^{-}(X)=$ $\varphi(X) \backslash X)$. 
Another interesting property of Minkowski addition for the construction of structuring elements is the following semi-group law: $n B \oplus m B=(n+m) B \Leftrightarrow$ $B$ is compact convex, where $n B$ is the homothetic factor $n$ of the structuring element $B$. The disks and the lines are compact convex sets since iteration acts as a magnification factor of structuring element. For instance, the dilation (resp. the erosion) by a segment of size $l$ and slope $\theta$ reduces to $l$ dilations (resp. erosions) of a segment of size 1.

\subsection{Theoretical generalization to the spatially-variant framework}

As detailed above, operators in mathematical morphology are in their classical definitions translation invariant mappings. However, in this study we need a more generic formulation where the operators will be variant with respect to translation in the space. That means that a different structuring element could be applied at each point and the final operation should verify the properties of standard operators.

The following classical results from Serra [1] for the Boolean lattice are needed. Every class $\mathcal{B} \subset \mathcal{P}(E)$ stable under the union and including $\varnothing$ characterizes an opening $\gamma$ on $\mathcal{P}(E)$ by the relation

$$
\gamma(X)=\cup\{B \mid B \subseteq X \text { and } B \in \mathcal{B}\} .
$$

The family $\mathcal{B}$ is composed of the invariant elements of the opening. It often acts by means of the generator $\mathcal{B}_{0} \subseteq \mathcal{B}$ which does not need to be stable with respect to the union. Consequently, $\mathcal{B}_{0}$ can be replaced by $\mathcal{B}$ in (6). That corresponds for instance to the circular disk of radius $r, C^{r}$, in the Euclidean case. The dual operation to $\gamma$ with respect to the complement is the closing $\varphi$ such as

$$
\varphi(X)=\gamma\left[\left(X^{c}\right)\right]^{c} .
$$

When the invariants $\mathcal{B}_{0}$ can be indexed by the points of the space $E$, i.e., to associate each point $x \in E$ with a corresponding unique $B(x)$, and that indexes all the elements of the generator $\mathcal{B}_{0}$ of $\mathcal{B}$, then, the opening $\gamma$ can be decomposed in an erosion $\varepsilon$ followed by its adjunct dilatation $\delta$, with

$$
\varepsilon(X)=\cup\left\{x \mid B(x) \subseteq X ; B(x) \in \mathcal{B}_{0}\right\}
$$

and

$$
\delta(X)=\cup\left\{B(x) \mid x \in X ; B(x) \in \mathcal{B}_{0}\right\} .
$$

Indeed, the following expression is obtained

$$
\delta \varepsilon(X)=\cup\left\{B(x) \mid x \in \varepsilon(X) ; B(x) \in \mathcal{B}_{0}\right\}=\cup\left\{B(x) \mid B(x) \subseteq X ; B(x) \in \mathcal{B}_{0}\right\},
$$

which corresponds exactly with (6). Both operations, $\delta$ and $\varepsilon$, are linked by the following duality "by adjunction'

$$
\delta(X) \subseteq Y \quad \Leftrightarrow \quad X \subseteq \varepsilon(Y) .
$$


Inversely, any pair of operators $(\varepsilon, \delta)$ which verifies $(7)$ will characterize an erosion and a dilation. As we can observe, this duality is very specific and different of the duality by the complement which is general for any operator on $\mathcal{P}(E)$.

The dual of $\varepsilon$ by the complement is $\delta^{*}$, where $\delta^{*}(X)=\left[\varepsilon\left(X^{c}\right)\right]^{c}$ is composed of the points $y$ of $E$ such that $B(y)$ hits $X$. If $X$ is reduced to one point, we have

$$
y \in \delta^{*}(x) \Leftrightarrow x \in B(y)=\delta(y) .
$$

Both dualities (7) and (8) are independent notions and they can not be expressed one as function of the other, at least without additional hypothesis. This last is the case for translation invariant in $E=\mathbb{R}^{n}$ or $\mathbb{Z}^{n}$, where (8) shows that $\delta(x)$ and $\delta^{*}(x)$ are then symmetrical with respect to the origin.

\subsection{Practical definitions of spatially-variant operators}

Let $\left\langle B_{\mathbf{x}}\right\rangle$ be the local structuring element for the point $\mathbf{x} \in E,\left\langle B_{\mathbf{x}}\right\rangle: E \rightarrow \mathcal{P}(E)$. A mapping $\mathbf{x} \rightarrow\left\langle B_{\mathbf{x}}\right\rangle$ is necessary for each point of $E$. Now a translation by vector $\mathbf{h}$ of the image, $X_{\mathbf{h}}$, involves the same translation for the structuring element at each point $\left.\left\langle B_{\mathbf{x}}\right\rangle\right|_{\mathbf{h}}$ in order to preserve the result of the operation.

Using the results of previous paragraphs, it is obvious that we can generalize the definition of dilation given in (1), in order to introduce the spatially-variant Boolean dilation by $\mathfrak{B}$, denoted by $\tilde{\delta}_{\left\langle B_{\mathbf{x}}\right\rangle}$, which can be written as:

$$
\tilde{\delta}_{\left\langle B_{\mathbf{x}}\right\rangle}(X)=\bigcup_{\mathbf{x} \in X}\left\langle\check{B}_{\mathbf{x}}\right\rangle .
$$

Using duality, the corresponding spatially-variant Boolean erosion, denoted by $\tilde{\varepsilon}_{\left\langle B_{\mathbf{x}}\right\rangle}$, is given by

$$
\tilde{\varepsilon}_{\left\langle B_{\mathbf{x}}\right\rangle}(X)=\left[\tilde{\delta}_{\left\langle B_{\mathbf{x}}\right\rangle}\left(X^{c}\right)\right]^{c} .
$$

Starting from the adjunction $\left(\tilde{\delta}_{\left\langle B_{\mathbf{x}}\right\rangle}, \tilde{\varepsilon}_{\left\langle B_{\mathbf{x}}\right\rangle}\right)$, the associated spatially-variant Boolean opening and closing are

$$
\tilde{\gamma}_{\left\langle B_{\mathbf{x}}\right\rangle}(X)=\bigcup_{\mathbf{x} \in E}\left\{\left\langle B_{\mathbf{x}}\right\rangle \mid\left\langle B_{\mathbf{x}}\right\rangle \subseteq X\right\},
$$

and

$$
\tilde{\varphi}_{\left\langle B_{\mathbf{x}}\right\rangle}(X)=\left[\tilde{\gamma}_{\left\langle B_{\mathbf{x}}\right\rangle}\left(X^{c}\right)\right]^{c} .
$$

It is obvious that all these spatially-variant Boolean operators verify the same properties than translation invariant ones. However, it should be remarked that the implementation of the opening (and the dual closing) needs to verify at each point $\mathbf{x}$ if its structuring element is totally contained in the set $X$.

\section{Directional field}

In this section, we focus our discussion on the orientation field estimation of a binary image, although the method introduced here can be applied to gray-level images. Firstly we revise the obtaining of the average squared gradient, for in a second stage, diffusing its vector field to all pixels of the image. 


\subsection{Average Squared gradient}

In order to obtain the orientation of the data in the images, several techniques based on the gradient have been studied. The use of the gradient and gradient vector flow (GVF) $[10,11]$ was discarded because it does not provide the main orientation of the data. The estimation of the orientation is obtained by using the average squared gradient (ASG) and the directional field used for spatially variant mathematical morphology is obtained by a diffusion process of the ASG field, the ASGVF. Fig 1 shows the vector fields provided by the gradient, GVF, $\mathrm{ASG}$ and ASGVF.

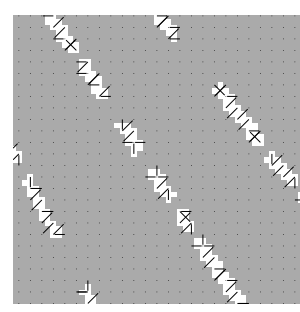

(a)

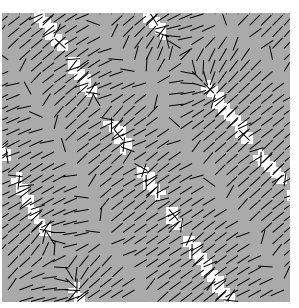

(b)

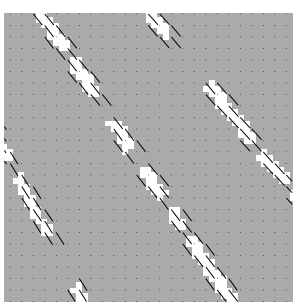

(c)

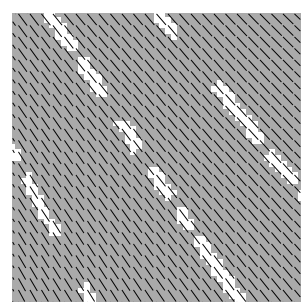

(d)

Fig. 1. Example of vector fields: (a) gradient, (b) gradient vector flow, (c) average squared gradient, (d) average squared gradient vector flow.

The average squared gradient (ASG) method provides the directional field by squaring and averaging the gradient vectors [12], [13]. Given an image $X(x, y)$, ASG uses the following definition of gradient

$$
\mathbf{g}=\left[\begin{array}{l}
g_{1}(x, y) \\
g_{2}(x, y)
\end{array}\right]=\operatorname{sign}\left(\frac{\partial X(x, y)}{\partial x}\right)\left[\begin{array}{l}
\frac{\partial X(x, y)}{\partial x} \\
\frac{\partial X(x, y)}{\partial y}
\end{array}\right] .
$$

Then the gradient is squared (doubling its angle and squaring its magnitude) and averaged in some neighborhood using the window $W$ :

$$
\overline{\mathbf{g}_{s}}=\left[\begin{array}{l}
\overline{g_{s, 1}}(x, y) \\
\overline{g_{s, 2}}(x, y)
\end{array}\right]=\left[\begin{array}{l}
\sum_{W}\left(g_{1}^{2}(x, y)-g_{2}^{2}(x, y)\right) \\
\sum_{W}\left(2 g_{1}(x, y) g_{2}(x, y)\right)
\end{array}\right] .
$$

The directional field ASG is $\mathbf{d}=\left[d_{1}(x, y), d_{2}(x, y)\right]^{\top}$, where its angle is obtained as

$$
\angle \mathbf{d}=\frac{\Phi}{2}-\operatorname{sign}(\Phi) \frac{\pi}{2}
$$

which is in the range $\left[-\frac{\pi}{2}, \frac{\pi}{2}\right]$, being $\Phi=\angle \overline{\mathbf{g}_{s}}$; and the magnitude of $\mathbf{d}$, $\|\mathbf{d}\|$, can be left as the magnitude of $\overline{\mathbf{g}_{s}}$, or the squared root of $\overline{\mathbf{g}_{s}}$ or, in some applications (see e.g [14]) and in this work, it can be set to unity. 


\subsection{Diffused Average Squared gradient}

The ASG field of an image has vectors pointing toward the orientation of the edges, which are parallel (tangent) to the edges at the edges. These vectors are generally different from zero only near the edges and, in homogeneous regions, where the gradient is nearly zero, the ASG is also zero (see Fig. 1(c)). In order to extend the orientation information to pixels where the gradient is nearly zero a diffusion process is performed, providing the ASG vector flow (ASGVF). The ASGVF is the vector field $\mathbf{v}=\left[v_{1}(x, y), v_{2}(x, y)\right]^{\top}$ that minimizes the energy functional:

$$
\mathcal{E}(\mathbf{v})=\mathcal{D}(\mathbf{v})+\alpha \mathcal{S}(\mathbf{v}),
$$

where $\mathcal{D}$ represents a distance measure given by the squared difference between the original and the regularized average squared gradient, weighted by the squared value of the last one,

$$
\mathcal{D}(\mathbf{v})=\frac{1}{2} \int_{E}\|\mathbf{d}\|^{2}\|\mathbf{v}-\mathbf{d}\|^{2} d x d y,
$$

where $E$ is the image support; the energy term $\mathcal{S}$ determines the smoothness of the directional field and represents the energy of the first order derivatives of the signal:

$$
\mathcal{S}(\mathbf{v})=\frac{1}{2} \int_{E}\left(\left(\frac{\partial v_{1}}{\partial x}\right)^{2}+\left(\frac{\partial v_{1}}{\partial y}\right)^{2}+\left(\frac{\partial v_{2}}{\partial x}\right)^{2}+\left(\frac{\partial v_{2}}{\partial y}\right)^{2}\right) d x d y
$$

This variational formulation produces a result which is smooth where the ASG is zero (there is no data). When the magnitude $\|\mathbf{d}\|$ of ASG is small, the energy is dominated by the sum of the squares of the partial derivatives of the vector field, yielding a slowly varying field, i.e., forcing the field to be slowlyvarying in homogeneous regions. On the other hand, when the magnitude of ASG is large, the first term dominates the functional (16) and the energy is minimized by setting the ASGF equal to ASG, i.e., producing the desired effect of keeping nearly equal to the ASG. The parameter $\alpha$ is a regularization parameter which governs the trade-off between the smoothness and data-fidelity.

Using the calculus of variations, the ASGF field can be found by solving the following Euler equations

$$
\begin{aligned}
& \left(v_{1}-d_{1}\right)\left(d_{1}^{2}+d_{2}^{2}\right)-\alpha \nabla^{2} v_{1}=0 \\
& \left(v_{2}-d_{1}\right)\left(d_{1}^{2}+d_{2}^{2}\right)-\alpha \nabla^{2} v_{2}=0
\end{aligned}
$$

or, more compactly

$$
(\mathbf{v}-\mathbf{d})|\mathbf{d}|^{2}-\alpha \nabla^{2} \mathbf{v}=\mathbf{0} .
$$

These equations can be solved by treating $\mathbf{v}$ as a function of time and considering the steady-state solution (which is equivalent to use the steepest descent method):

$$
\mathbf{v}_{t}+(\mathbf{v}-\mathbf{d})|\mathbf{d}|^{2}-\alpha \nabla^{2} \mathbf{v}=\mathbf{0} .
$$


These equations are known as generalized diffusion equations. To set up the iterative solution, let the indices $i, j$, and $n$ correspond to $x, y$ and $t$, respectively, and let the spacing between pixels be $\Delta x$ and $\Delta y$, and the time step for each iteration be $\Delta t$. Then the required partial derivatives can be approximated as

$$
\begin{aligned}
\mathbf{v}_{t} & \approx \frac{\mathbf{v}^{n}-\mathbf{v}^{n-1}}{\Delta t} \\
\nabla^{2} \mathbf{v}_{i, j} & \approx \frac{\mathbf{v}_{i-1, j}+\mathbf{v}_{i+1, j}-2 \mathbf{v}_{i, j}}{\Delta x^{2}}+\frac{\mathbf{v}_{i, j-1}+\mathbf{v}_{i, j+1}-2 \mathbf{v}_{i, j}}{\Delta y^{2}}
\end{aligned}
$$

Considering discrete images $(\Delta x=\Delta y=1)$ and substituting these approximations into the equations gives our iterative solution to ASGF as follows:

$$
v_{i, j}^{l, n}=v_{i, j}^{l, n-1}-\Delta t f_{i, j}^{l, n-1}+\frac{1}{\eta}\left(v_{i+1, j}^{l, n-1}+v_{i-1, j}^{l, n-1}+v_{i, j+1}^{l, n-1}+v_{i, j-1}^{l, n-1}-4 v_{i, j}^{l, n-1}\right)
$$

where $f=\left(v_{i, j}^{l, n-1}-d_{i, j}^{l}\right)\left|\mathbf{d}_{i, j}\right|^{2}, l$ is the component index $l=1,2, n$ is the iteration index and $\eta=(\alpha \Delta t)^{-1}$.

\section{Results}

We have now associated to each 2D image $X \in \mathcal{P}(E), E \subset \mathbb{Z}^{2}, \mathbf{x}=(x, y) \in E$, a regularized directional vectorial field, i.e., $\mathbf{F}^{\theta}(\mathbf{x})=\left(v_{1}(\mathbf{x}), v_{2}(\mathbf{x})\right)$, where the vectorial field $\mathbf{F}^{\theta}(\mathbf{x})$ is defined in $E \times \mathbb{Z}^{2}$ space. Let us consider in fact that the vectorial field defines a scalar value to each point which corresponds to the orientation of the data at point $\mathbf{x}$, i.e., $\theta(\mathbf{x})$. The corresponding local structuring element (SE) of a linear segment of size $l$ and orientation $\theta(\mathbf{x})$ is denoted $\left\langle L_{\mathbf{x}}^{l, \theta_{\mathbf{x}}}\right\rangle$. Note that in the examples given in this paper the length of the local structuring element is constant for all the points, but we can also consider spatially variable lengths.

We have included three examples of the application of locally orientated morphological operator in order to regularize binary objects: to filter out structures of small length and to reconnected close linear structures. Two first examples are given in Fig. 2 and Fig. 3 (size $256 \times 256$ pixels. The average squared gradient has been obtained using a flat squared averaging window of size $19 \times 19$. The value of the constants for the diffusion process are $\Delta t=1$ and $\eta=10^{-3}$. In both cases, the length of the SE for all the morphological filters is $l=11$. A more complex case study is depicted in Fig. $4(404 \times 599$ pixels $)$. The average squared gradient has been obtained using a flat squared averaging window of size $27 \times 27$ and value of the constant equals $\eta=10^{-1}$. The length of the structuring element is now $l=15$.

One aspect to take into consideration is the size of the SE. A trade-off between size and angular resolution must be reached. Small SE allows get into small details of the objects but offers few directions. Bigger SE can not deal with these small details but offer many directions. In summary, if the linear SE is $N$ pixel long, the angular resolution is $\Delta \alpha=\frac{90}{N-1}$ degrees, the discretized angles being $\alpha_{i}=\Delta \alpha i, \quad i \in[0,2(N-1)-1]$, as shown in Fig. 5 for $N=11$. 


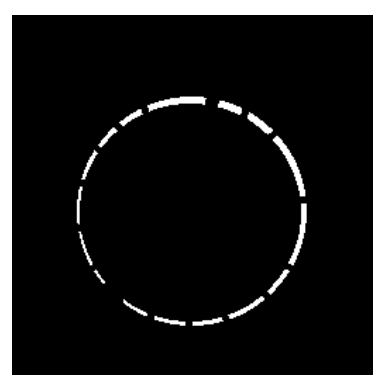

(a) $X$

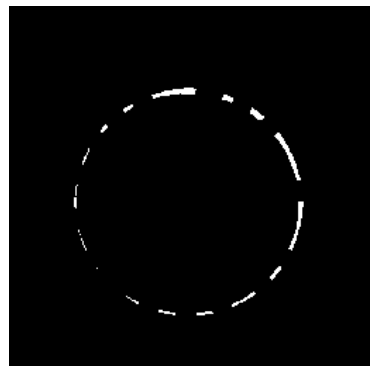

(c) $\tilde{\varepsilon}_{\left\langle B_{\mathbf{x}}\right\rangle}(X)$

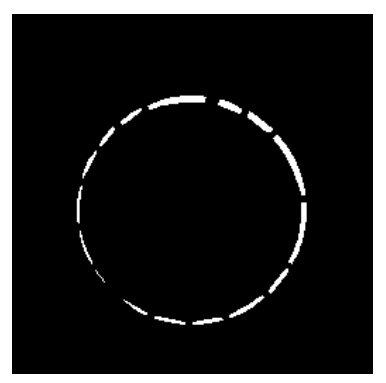

(e) $\tilde{\gamma}_{\left\langle B_{\mathbf{x}}\right\rangle}(X)$

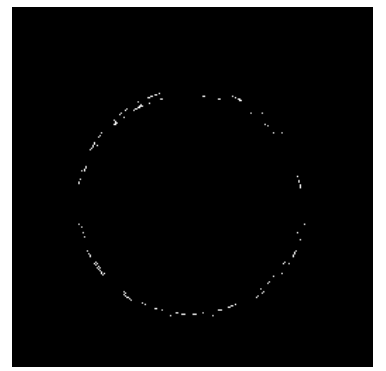

(g) $t h^{+}(X)$

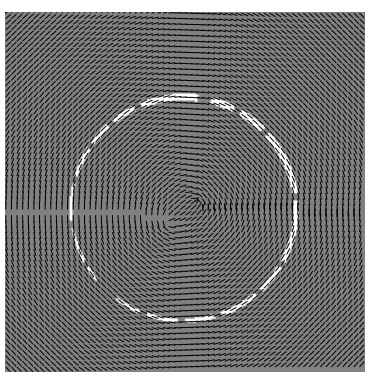

(b) ASGV field

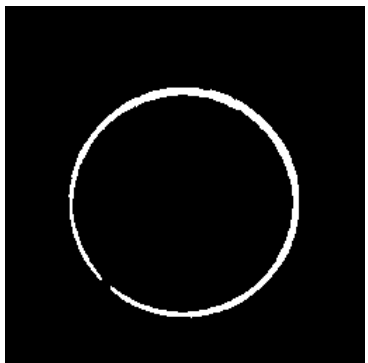

(d) $\tilde{\delta}_{\left\langle B_{\mathbf{x}}\right\rangle}(X)$

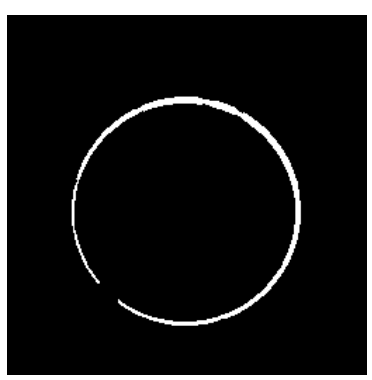

(f) $\tilde{\varphi}_{\left\langle B_{\mathbf{x}}\right\rangle}(X)$

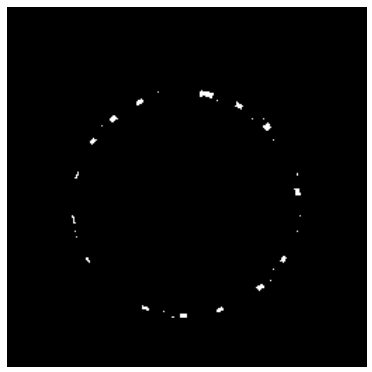

(h) $t h^{-}(X)$

Fig. 2. (a) Original image $256 \times 256$ pixels, (b) ASGV field using $\eta=0.001$, (c) erosion with a orientated linear structuring element with length 7 pixels, (d) dilation with the same structuring element, (e) opening, (f) closing, (g) top-hat and (h) dual top-hat. 


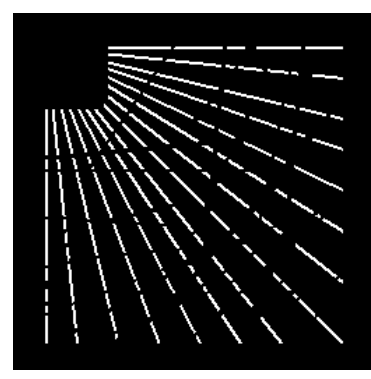

(a) $f(\mathbf{x})$

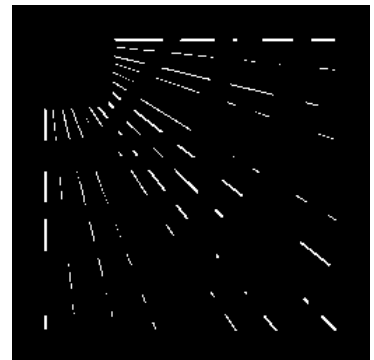

(c) $\tilde{\varepsilon}_{\left\langle B_{\mathbf{x}}\right\rangle}(X)$

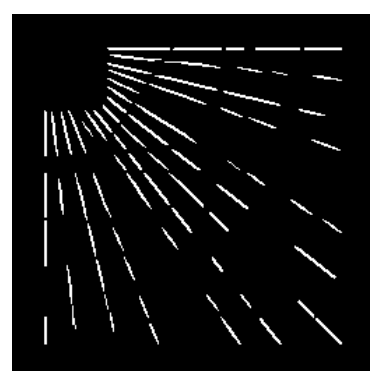

(e) $\tilde{\gamma}_{\left\langle B_{\mathbf{x}}\right\rangle}(X)$

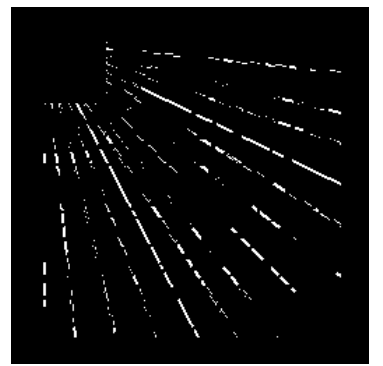

(g) $t h^{+}(X)$

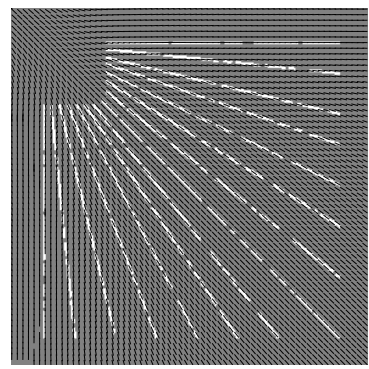

(b) ASGV field

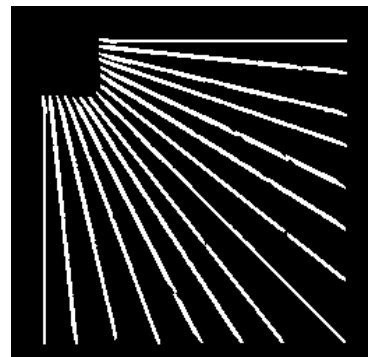

(d) $\tilde{\delta}_{\left\langle B_{\mathbf{x}}\right\rangle}(X)$

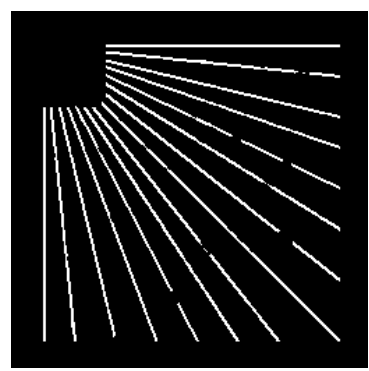

(f) $\tilde{\varphi}_{\left\langle B_{\mathbf{x}}\right\rangle}(X)$

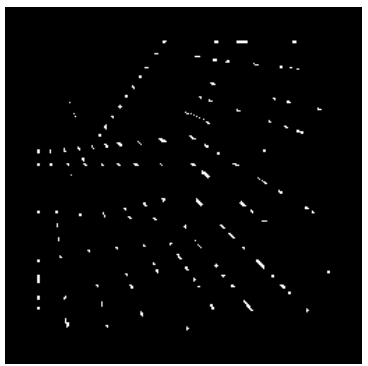

(h) $t h^{-}(X)$

Fig. 3. (a) Original image $256 \times 256$ pixels, (b) ASGV field using $\eta=0.001$, (c) erosion with a orientated linear structuring element with length 7 pixels, (d) dilation with the same structuring element, (e) opening, (f) closing, (g) top-hat and (h) dual top-hat. 


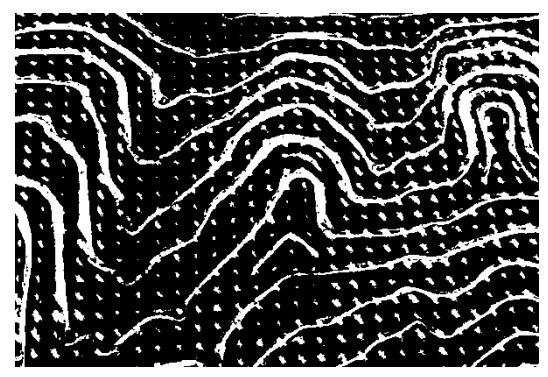

(a) $f(\mathbf{x})$

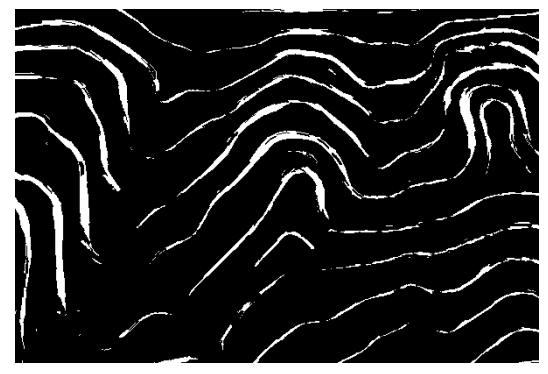

(c) $\tilde{\varepsilon}_{\left\langle B_{\mathbf{x}}\right\rangle}(X)$

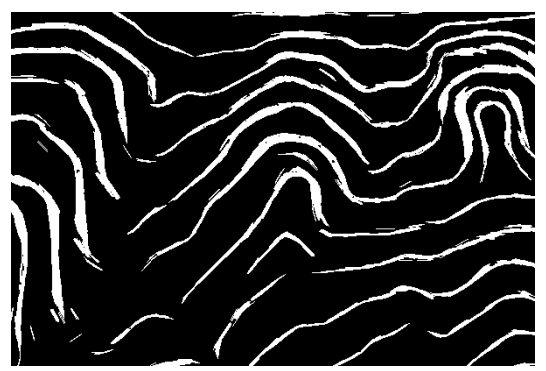

(e) $\tilde{\gamma}_{\left\langle B_{\mathbf{x}}\right\rangle}(X)$

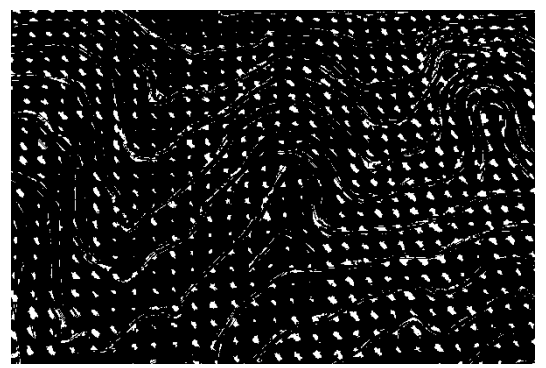

(g) $t h^{+}(X)$

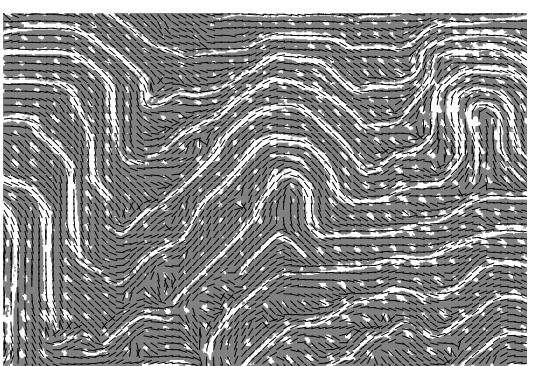

(b) ASGV field

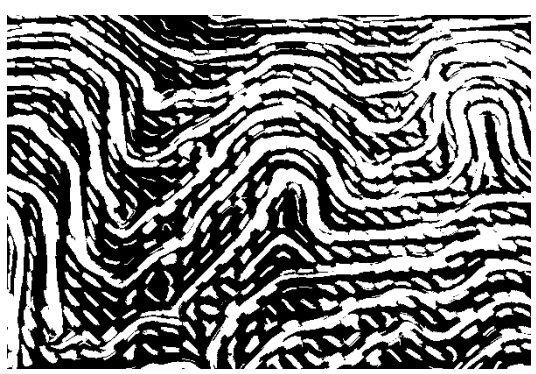

(d) $\tilde{\delta}_{\left\langle B_{\mathbf{x}}\right\rangle}(X)$

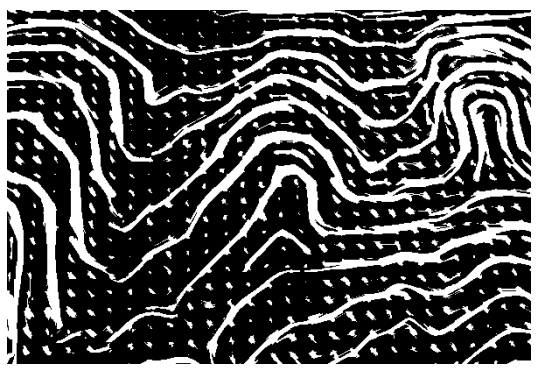

(f) $\tilde{\varphi}_{\left\langle B_{\mathbf{x}}\right\rangle}(X)$

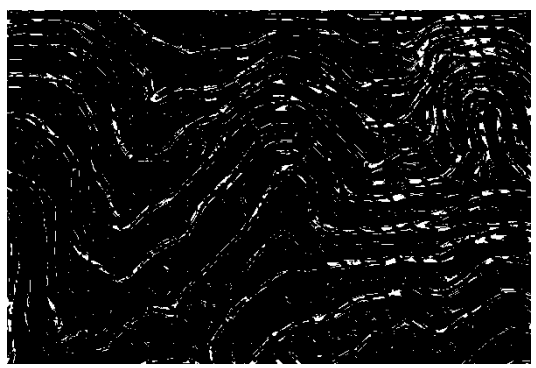

(h) $t h^{-}(X)$

Fig. 4. (a) Original image $404 \times 599$ pixels, (b) ASGV field using $\eta=0.1$, (c) erosion with a orientated linear structuring element with length 15 pixels, (d) dilation with the same structuring element, (e) opening, (f) closing, (g) top-hat and (h) dual top-hat. 
Fig. 5. Angular discretization using a linear SE with 11 pixels.

\section{Conclusions}

After reminding the theoretical elements of spatially-variant mathematical morphology, a new approach of regularization of the directional field of an image has been described. We have then proved the interest of linear openings for reconnecting objects of an image described locally by directional information. This problem concerns many image processing applications, for example, in medicine, biometrics, metallurgy and geology.

As future works, we intend to generalize our approach to other families of local anisotropic structuring elements (i.e., ellipses) and to extend the algorithms to grey level images. The theoretical extension to gray level images, and above all, the optimized implementation of spatially variant dilations and openings is still an open field.

\section{References}

1. Serra, J.: Image Analysis and Mathematical Morphology: Theoretical Advances. Vol. II. Academic Press, London (1988)

2. Soille, P., Talbot, H: Directional morphological filtering. PAMI 23(2001)1313-1329

3. Heijmans, H., Buckley, M., Talbot, H.: Path openings and closings. Journal of Mathematical Imaging and Vision 22 (2005) 107-119

4. Bouaynaya, N., Schonfeld, D.: Theoretical foundations of spatially-variant mathematical morphology part ii: Gray-level images. PAMI 30 (2008) 837-850

5. Roerdink, J.: Group morphology. Pattern Recognition 33 (2000) 877-895

6. Cuisenaire, O.: Locally adaptable mathematical morphology using distance transformations. Pattern Recognition 39 (2006) 405-416

7. Lerallut, R., Decenciere, E., Meyer, F.: Image filtering using morphological amoebas. Image Vision Comput. 25 (2007) 395-404

8. Serra, J.: Image Analysis and Mathematical Morphology. Vol. I. (1982)

9. Soille, P.: Morphological Image Analysis. Springer-Verlag (1999)

10. Xu, C., Prince, J.L.: Generalized gradient vector flow external forces for active contours. Signal Processing 71 (1998) 131-139

11. Xu, C., Prince, J.L.: Snakes, shapes, and gradient vector flow. IEEE Trans. Image Processing 7 (1998) 359-369

12. Kass, M., Witkin, A.: Analyzing oriented patterns. Comput. Vision Graph. Image Process. 37 (1987) 362-385

13. Bazen, A.M., Gerez, S.H.: Systematic methods for the computation of the directional fields and singular points of fingerprints. PAMI 24 (2002) 905-919

14. Perona, P.: Orientation diffusions. IEEE Trans. Image Processing 7 (1998) 457-467 